\title{
实验动物树鼠句和人类疾病的树鼠模型研究概述
}

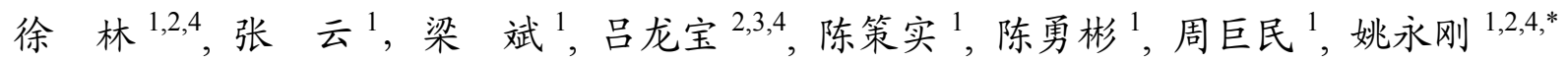

1. 中国科学院和云南省动物模型与人类疾病机理重点实验室, 中国科学院昆明动物研究所 云南 昆明 650223 ;

2. 中国科学院昆明动物研究所, 中国科学院灵长类研究中心, 云南 昆明 650223 ;

3. 中国科学院昆明动物研究所实验动物中心, 云南 昆明 650223 ;

4. 中国科学院树鼠繁殖基地, 云南 昆明 650223

摘要: 动物模型在生物医学领域（如回答人体各种重大生物学问题、解析人类疾病机理和新药研发等方面）已经做出了不可 替代的巨大贡献。转化医学存在的问题使得树鼠 (Tupaia belangeri chinensis) 实验动物重新得到重视; 人类疾病的树舄模型 也再次受到越来越多的关注。该文综述了国内外特别是近年来我国树舀研究进展, 包括树舄基础生物学及动物模型方面取得 的成绩，并分析了该领域目前存在的困难和问题，探讨了未来的一些研究方向。

关键词: 树鼠; 实验动物; 基础生物学; 人类疾病动物模型

中图分类号: Q4; Q95-331 文献标志码: A 文章编号: 0254-5853-(2013)02-0059-11

\section{Tree shrews under the spot light: emerging model of human diseases}

Lin $\mathrm{XU}^{1,2,4}$, Yun ZHANG ${ }^{1}$, Bin $\mathrm{LIANG}^{1}$, Long-Bao $\mathrm{LÜ}^{2,3}$, Ce-Shi CHEN ${ }^{1}$, Yong-Bin CHEN ${ }^{1}$, Ju-Min ZHOU ${ }^{1}$, Yong-Gang YAO ${ }^{1,2,4, *}$

1. Key Laboratory of Animal Models and Human Disease Mechanisms of the Chinese Academy of Sciences \& Yunnan Province, Kunming Institute of Zoology, Kunming 650223, China;

2. Kunming Primate Research Center, Kunming Institute of Zoology, the Chinese Academy of Sciences, Kunming 650223, China;

3. Experimental Animal Core Facility \& Kunming Primate Research Center, Kunming Institute of Zoology, the Chinese Academy of Sciences, Kunming 650223, China ;

4. Tree Shrew Inbreeding Center, Kunming Institute of Zoology, the Chinese Academy of Sciences, Kunming 650223, China

Abstract: Animal models are indispensible in biomedical research and have made tremendous contributions to answer fundamental questions on human biology, disease mechanisms, and to the development of new drugs and diagnostic tools. Due to the limitations of rodent models in translational medicine, tree shrews (Tupaia belangeri chinensis), the closest relative of primates, have attracted increasing attention in modeling human diseases and therapeutic responses. Here we discuss the recent progress in tree shrew biology and the development of tree shrews as human disease models including infectious diseases, metabolic diseases, neurological and psychiatric diseases, and cancers. Meanwhile, the current problems and future perspectives of the tree shrew model are explored.

Keywords: Tree shrew; Experimental animals; Fundamental biology; Animal models of human diseases

\section{1 前 言}

\section{1 动物模型和模式动物的概念}

人体是一个极其复杂的系统。科学家利用简单 的原生动物到复杂的非人灵长类动物, 为解析人体 各种复杂的生物学问题和疾病机理进行了长期不 懈的努力。动物模型的概念即为利用动物创建模型 去模拟人体各种复杂的生物学问题以及疾病特征
和过程, 进而研究其基本规律和分子细胞机理。在 动物模型的概念基础上, 逐渐形成了模式动物的概 念。为了使动物模型实验具有重复性、稳定性、可 比性, 以及符合动物伦理等要求, 建立遗传背景清 楚、稳定, 饲养繁殖环境条件符合法规的动物品系 就成为必要条件, 这就是模式动物的概念。利用模 式动物创建的动物模型, 能更好地研究人体各种复 杂的生物学问题、疾病机理及治疗手段 $(\mathrm{Xu}, 2011)$ 。

收稿日期: 2012-12-15; 接受日期: 2012-12-30

基金项目: 中国科学院基础前沿研究专项项目(KSCX2-EW-J-23); 863 课题(2012AA021801); 中国科学院知识创新工程重要方向项目 (KSCX2-EW-R-11, KSCX2-EW-R-12)

*通信作者(Corresponding author), E-mail: yaoyg@mail.kiz.ac.cn 


\section{2 动物模型的重要性}

尽管早期没有成熟的模式动物, 纵观 100 多年 的诺贝尔生理学或医学奖, 仍可清晰地看到动物模 型在生物医学领域中的重要地位。1901-2012 年, 共有 201 位科学家获得诺贝尔生理学或医学奖 (http://www.nobelprize.org/nobel_prizes/medicine/lau reates/index.html), 其中 166 位的科研成果来自于动 物模型实验, 占诺贝尔生理学或医学奖的 $83 \%$ 。特 别是在过去的 40 年间 (1973-2012 年), 94 位获得 诺贝尔生理学或医学奖的科学家中, 仅 Barbara McClintock 博士因植物遗传学(转座子理论) 获奖 (1983 年), 其他 93 位 (99\%)科学家的获奖均得益于 动物模型实验。这些重大生物医学成果涉及的动物 种类包括原生动物、线虫、软体动物、甲壳动物、 果蝇、鱼类、鸟类、两栖类、爬行类、啮齿类 (大、 小鼠)、兔、猪、牛、羊、马、以及非人灵长类等(图 $1)$ 。许多诺贝尔生理学或医学奖获得者使用多种动 物, 因为每种动物具有各自的优缺点, 对解决特定 问题具有特殊的优势。由于伦理及其他问题, 如可 控性和方便操作等, 许多基于人类疾病机理解析和 药物研发的实验只能依赖于动物模型的建立和研 究 $(X u, 2011)$ 。

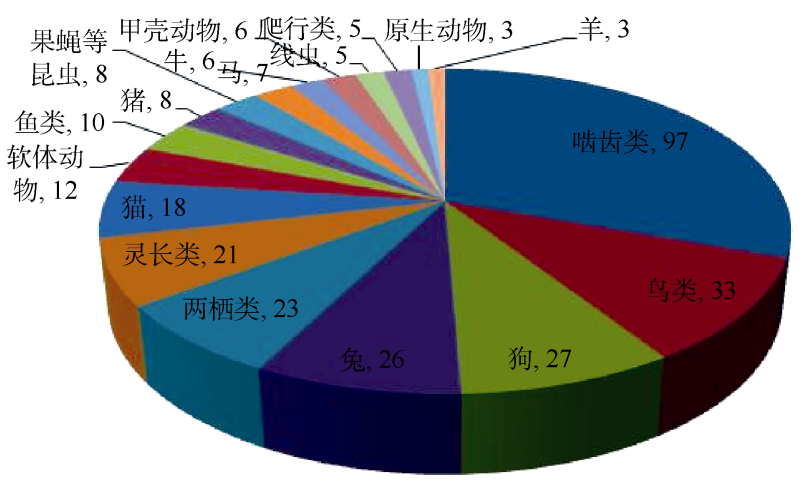

图 1 使用不同种类动物的诺贝尔生理学和医学奖获奖人数 Figure 1 Number of Noble laureates and animal models used

\section{3 动物模型的最佳策略}

随着基因组学、表观基因组学、转录组学、蛋 白质组学和代谢组学等研究的迅猛发展, 各种动物 在生物医学研究领域中的地位正经历着剧变, 研究 者已经逐渐清楚认识利用不同动物模型回答人体 各种重大生物问题和疾病机理的优缺点。总的来 说, 低等动物的优点是实验周期短、成本低及基因 操作方便等; 缺点是与人类的差异性较大。相反, 高等动物如非人灵长类与人类的差异性小, 研究结
果很容易直接转化为临床应用; 缺点是实验周期 长，成本高且基因操作困难等。

任何一种有机体即使是简单的原生动物也是 一个极其复杂的生命系统，而人类的生命系统尤其 是大脑已经演化成自然界最复杂的系统。然而, 生 命现象在分子细胞层次上却仍然遵循着相似或相 同的最基本规律。因此，如果想回答人体各种重大 的生物学问题和疾病的分子细胞机理, 使用低等动 物显然具有独特优势; 相反，如果想回答各种分子 细胞机理是否在整体水平上表现类似于人类的生 物学问题以及疾病特征和过程, 则必须依赖高等 动物。

值得特别关注的是，其他动物（如广泛使用的 啮齿类）在分子细胞层次上能提供极大帮助，但研 究人类特有的疾病以及智力和创造力, 则使用非人 灵长类动物在整体水平上具有不可替代的优势。因 而, 理解人体各种重大生物问题和疾病机理的最佳 策略有两个选择: 1)低等动物的分子细胞机理研究 和高等非人灵长类动物的整体系统水平研究相结 合；2)寻找新型动物模型，兼顾成本低、试验周期 短、分子和细胞操作方便并与人类进化地位接近等 各种生物学特性。前者已被许多诺贝尔生理学或医 学奖获得者采用, 而后者正在被科学家和生物医药 领域采用, 如树鼠(Tupaia belangeri chinensis)即为 后者最典型的动物。

树鼠被认为是灵长类最亲密的近亲, 已有证据 表明树鼠非常适合用于研究人体的近视、心理应激 和肝炎等问题(Cao et al，2003; Wang et al，2012)。 作为实验动物, 树鼠在生命医学领域已被使用 30 多年。本文重点归纳了国内外, 特别是近年来我国 在树鼠基础生物学及疾病动物模型研制方面取得 的进展，分析和讨论了该领域存在的困难和未来的 研究方向。

\section{2 树鼠基础生物学}

\section{1 树鼠简介}

树鼠属攀鼠目(Scandentia)树鼠科(Tupaiidae)。 我国现生树鼠被认为有 1 属 1 种 6 个亚种, 即中缅 树䳔 (Tupaia belangeri) 1 个种, 6 个亚种 (Peng et al, 1991; Wang，1987)。云南是野生树舀分布的主 要区域之一, 拥有 3 个亚种 (Wang, 1987)。树鼠 体型小，与大鼠类似，在人工繁殖条件下无明显繁 殖季节，孕期短，生殖与发育周期短，出生后 $4 \sim 6$ 
个月就达到成年期, 每胎 $2 \sim 6$ 只。

中国科学院昆明动物研究所是国内最早开展 树鼠研究的单位, 早在 1991 年就出版了国内第一 本也是目前仅有的一本《树鼠生物学》。专著系统 总结了树鼠生物学特性, 包括树鼠分类与生态学、 饲养与繁殖、寄生虫学与病理学、解剖学、神经生 物学、遗传学、生物化学和免疫学等 (Peng et al, 1991)。该专著出版后, 陆续也有学者发表了一些树 鼠生物学基础数据, 如近期 Ma et al (2011)研究比较 了野生和人工繁育树鼠的部分生理指标, 发现在两 组动物中肌酸激酶、肌钙蛋白 I、总胆汁酸、果糖 胺及低密度脂蛋白胆固醇等生理生化指标在性别 间无显著差异, 而在野生和人工繁育个体中存在差 异, 且野生个体间差异较大。Wang et al (2013)研究 了树鼣体重、体温、进食量、尿量, 以及血液中各 种激素含量。Wu et al (2013)研究了 992 只树鼠体 重、空腹血糖、性别和年龄之间的关系, 发现其空 腹血糖随体重增加而上升, 且该相关性仅见于雄性 个体。雌性树鼠的体重、空腹血糖、腰围随年龄而 增加。树鼣体重、空腹血糖、性别和年龄之间的关 系与其他非人灵长类和人类非常相似。这些基础数 据的获得, 进一步丰富了我们对于树舀的认识。

最近, 昆明动物研究所组织了中缅树鼠全基因 组测定和解析工作, 得到了高覆盖度(79X)的基因组 序列。通过基因组分析, 发现树鼠在神经及免疫系 统等方面与人类具有较为高度的同源性, 在很多方 面具有可用来替代猕猴等大型灵长类实验动物的 遗传基础, 可能还适合于更多的免疫学、神经生物 学及代谢等生物学问题和疾病机理研究(Fan et al, 2012)。同时, 比较基因组分析发现, 树鼠药物代谢 酶与人体接近, 而狝猴则缺失一大家族的药物代谢 酶。Li RX et al (2012) 的研究表明, 树鼠的肝脏和 肌肉组织蛋白组表达谱较啮齿类动物大鼠和小鼠 更接近于人类。这些树獡资源优势和研究积累, 为 进一步发掘树鼠资源, 建立树鼣实验动物品系和模 型奠定了极好的基础。

\section{2 树鼠是灵长类近亲}

长期以来, 树獡与灵长类的亲缘关系一直存在 争论。尽管目前树䳔的具体系统发育分类地位仍有 争议, 但多数研究显示其与灵长类具有最接近的亲 缘关系(Xu et al, 2013)。基于不同类型的遗传学数 据, 得到的树鼠与灵长类关系也不尽相同 (Xu et al, 2013)。Adkins \& Honeycutt (1991)通过线粒体细
胞色素氧化酶 II (COX II) 将树鼠与其他 7 个哺乳动 物线粒体 COX II 序列进行系统发育划分, 证实树 鼠是与灵长目动物最近缘的动物。Xu et al (2012)及 Schmitz et al (2000)在构建线粒体 12 个蛋白编码基 因的系统发育树时, 发现树鼠与兔形目动物之间的 关系更加密切, 而皮翼目动物和灵长类动物组成姐 妹群。在利用多个核基因序列构树时依然出现了这 样不一致的结果 (Xu et al, 2013)。Janečka et al (2007) 根据 21 个灵长总目动物的基因组中罕见的 外显子插入缺失和长度约为 $14 \mathrm{~kb}$ 的 19 个核基因拼 接片段构建的系统树显示树鼠与灵长类的亲缘关 系更近。Lindblad-Toh et al (2011)利用美国 Broad 研 究所公布的 $2 X$ 的树鼣基因组数据重构 29 个哺乳动 物的系统发育关系, 树鼣是其中最接近灵长类的动 物。然而 Hallström \& Janke (2010)利用 3000 基因重 构系统发育树却显示树鼣与啮齿总目的亲缘关系 更近。基于染色体图染数据分析的结果显示树鼠目 与皮翼目形成姐妹群 (Nie et al, 2008)。昆明动物 所近期基于高质量的中缅树鼠的全基因组中 2117 个单拷贝基因重构了树䳔与 14 个哺乳类动物(其中 包括 6 个灵长类物种)的系统发育关系, 显示树鼠与 灵长类的亲缘关系更近, 解决了长久以来关于树䳔 进化地位的争议。在将楖舄和啮齿类的蛋白质序列 与人的同源基因进行一对一比较时, 发现人与树鼠 之间较人与啮齿类之间拥有更高的蛋白质相似度, 进一步说明树䳔与灵长类亲缘关系更近。同时发现 树䳔与灵长类在一些重要的神经系统和免疫系统 信号通路中拥有高度的同源性 (Fan et al，2012)。

\section{3 树鼠实验动物化和品系的创制}

由于受到资源的限制, 国际上饲养、繁殖树䳔 的中心不多, 仅有德国灵长类中心(Gottingen)、美 国伯明翰阿拉巴马大学、美国圣地亚哥野生动物 园、墨西哥沃尔夫繁殖中心和欧洲灵长类中心(荷兰) 等, 远少于灵长类饲养繁殖中心。国际大多数文献 中使用的树鼣来自德国灵长类中心。国内大多数文 献和国际部分文献中使用的树鼠来自中国科学院 昆明动物所实验动物中心。

我国从上世纪 70-80 年代开展树鼠人工驯养 繁殖及相关研究, 研究力量主要集中在昆明, 如中 国科学院昆明动物研究所、中国医学科学院医学生 物学研究所、昆明医科大学及云南省地方病防治研 究所等科研院校 (Shen et al, 2011)。昆明动物研究 所是我国开展树鼠人工驯养繁殖最早的单位, 通过 
对树鼣饲养环境及设施、微生物、寄生虫和配合饲 料等的不断探索改进, 已形成规范的饲养管理体 系, 繁育技术成熟, 实现了树鼠的标准化人工驯养 繁育, 并于 2012 年 10 月通过树鼠生产和使用许可 证认证, 成为全国首家获得树鼠生产许可证的单 位, 意味着树鼠将更容易被推广至全国乃至世界。 目前昆明动物研究所稳定的树舀饲养繁殖种群 3000 头, 每年为国内外几十家单位提供实验树鼠 1000 头; 人工饲养的封闭群树鼠已经达到子 6 代; “十二五”末预期繁殖饲养规模达到 4000 5000 头, 并育成近交系第 5 代。昆明医科大学、广西医科大 学、中国医学科学院医学生物学研究所等多家单位 也开展了树鼠的驯养繁殖工作(Shen et al, 2011), 并取得了不少成功经验。2010 年, 由昆明医学院调 研和编制, 云南省质量技术监督局颁布《实验树鼣 云南省地方标准》- - 《实验树鼠 (DB53/T 328.1-328.5-2010)》, 形成了实验动物树鼠的云南 省地方标准。

然而, 目前研究中使用的树鼠大多来自野外或 驯化后代, 它们的年龄和遗传背景很不清楚, 且个 体差异较大, 这使得利用树獡进行疾病机理研究和 新药创制困难重重。因此, 创建遗传背景清晰、稳 定的树鼠品系尤为重要。建立树鼠近交系是一项长 期且艰巨的任务, 目前国内外还未能培育出树鼠的 近交系。昆明动物研究所目前已就这一难题组织了 专门的团队进行攻关, 已经建立了 200 多个家系的 近交繁殖群体, 获得了兄弟姐妹近交 F2 代树獡。 同时, 为了更好地评估树鼣资源, 我们在前期还对 来自昆明郊区的树鼠群体进行了 mtDNA 遗传多样 性评估, 发现该树鼠群体具有较高的遗传多样性 (Chen et al，2011)。为配合树鼠近交系建立和评估 工作, 我们设计了一套可用于树鼣个体识别和亲权 鉴定分析的微卫星标记。采用该标记, 可达到 $>$ 99.99999998\%的个体区分识别能力。通过分析 117 只采自于云南省昆明周边的树鼣群体这套 12 个微 卫星座位的多态性, 显示了较高的期望杂合度 (0.616), 进一步支持我们 mtDNA 分析揭示的树鼠 群体高度多态性(Liu \& Yao, 2013)。

\section{3 树鼠动物模型研究现状}

树鼠作为灵长类动物的近亲, 在生理解剖、神
经发育、病毒感染特性及心理应激模式等方面与灵 长类甚至人类之间存在高度的相似性。在神经系统 方面, 树鼠的四爪对趾使其能够单爪抓握东西, 说 明其神经系统发育良好; 人类前额叶高度发育, 树 鼠与灵长类的大脑前额叶已经发育, 而大、小鼠等 的大脑前额叶几乎没有发育; 树鼠的大脑/体重比甚 至高于人类 (Peng et al，1991)。树鼠神经系统的这 些发育特点, 无疑为采用树舄作为人类神经系统疾 病的动物模型提供了坚实基础。同时，树鼠体表面 积与人体 $60 \mathrm{~kg}$ 的换算比例为 5.1 , 大鼠为 7 , 而小 鼠则高达 $14{ }^{1}$, 提示相对于大鼠、小鼠, 树鼠可更 好地应用于药物研究。另外, 树鼠的许多病毒感染 特性与人类相似，报道显示树鼠能感染人类甲肝、 乙肝、丙肝、轮状、疮疹、腺、棒状及副粘等病毒 (Han et al, 2011; Li et al，2011; Peng et al，1991; Wang et al, 2011)。

\section{1 感染性疾病树鼠模型}

\section{1 .1 病毒性肝炎模型}

除人类之外, 目前能自然感染人乙型、丙型肝 炎病毒的只有黑猩猩。但是因其资源贵乏、再加上 经济和道德伦理上的问题阻碍了黑猩猩模型的发 展。将肝炎病毒基因导入小鼠内的转基因模型不能 模拟病毒感染的过程。而人肝细胞嵌合小鼠(human hepatocyte chimeric mice)虽经证实能感染人乙型、 丙型肝炎病毒，但无法用于研究肝炎病毒感染的炎 症和免疫反应以及感染后期症状(如肝炎病毒感染 引起的肝癌)(Chayama et al, 2011)。

甲型肝炎(hepatitis A virus, HAV)模型 Zhan et al (1981)首次报道了利用树鼠作为动物模型, 用 甲肝病毒阳性病人的新鲜大便滤液感染动物, 证明 人的甲型肝炎病毒可以在树鼣体内繁殖, 但是否引 起树鼠肝病变尚无明确研究。

乙型肝炎(hepatitis B virus, HBV)模型 乙肝病 毒是一种 DNA 病毒, 属嗜肝 DNA 病毒科 (hepadnaviridae)。Pang et al (1981)报道了“乙型肝炎 病毒感染树鼠的实验研究”。随后, 广西肿瘤防治 研究所 Su et al (1986)用分子杂交、电镜、血清免疫 学和免疫组化等技术, 证实树鼣能够感染人的 HBV 病毒。后来广西医科大学肿瘤防治研究所 Yan et al (1984)用人血来源的 HBV 感染树鼣, 确定 10 只动 物中有 7 只感染。这些工作表明除人类、黑猩猩以 
外, 树鼠能感染人 HBV, 且具有成本低、实验操作 方便、不受动物伦理严格限制等独特优点。Walter et al (1996)通过体内外实验, 进一步证实树鼠可以作 为人 $\mathrm{HBV}$ 感染的研究模型。值得注意的是, Walter et al (1996)报道的树鼣新生期接种人 HBV, 结果类 似于人的急性自限性肝炎, 而树鼠成年期接种则表 现为一过性感染。Liang et al (2006) 用幼年和围生期 的树舄感染人 HBV, 揭示可以提高感染效率。

虽然国内外研究树䳔 HBV 感染已经有 20 多 年, 但树鼠体内感染能否长期持续仍存争议( $\mathrm{Li}$ et al, 2011)。Yang et al (2009)用新生树鼠感染人 HBV, 证实病毒能在动物体内长期存在并稳定复制。瑞士 的 Walter et al (1996)报道的树鼣体外感染人 HBV 的研究, 促进了 HBV 感染机理的研究。德国的 Glebe et al (2003)发现人 HBV 的 Pre-S1 抗原在感染树鼠 肝细胞中起重要作用。最近, 北京生命科学研究所 Yan et al (2012)根据已经报道的 HBV 结合受体的肽 段 Pre-S1 设计探针, 借鉴 Walter et al (1996)报道的 树鼠肝细胞体外培养方法, 以树鼠原代肝细胞 (primary Tupaia hepatocytes, PTH)为材料, 找到了乙 肝病毒和丁肝病毒的共用受体一一钠离子一牛磺胆 酸共转运多肽。这一发现将有力推动有效治疗乙肝 药物的研究和解析乙肝病毒感染机理。

丙型肝炎(hepatitis $\mathrm{C}$ virus, HCV)模型 丙型 肝炎的动物模型主要有黑猩猩、猴类、人工改造的 小鼠和树鼠 (Li et al, 2011)。Wang et al (1997)和 Liu et al (1998)用 HCV 阳性血清感染树鼠, 结果动 物出现不同程度的肝炎症状。Xie et al (1998)用 $\mathrm{HCV}$ 感染射线处理后的树鼣, 发现辐射后的树鼠对 HCV 更易感。Amako et al (2010)进行了长达 3 年的 $\mathrm{HCV}$ 感染树鼠研究, 进一步证实了树鼠可以作为 $\mathrm{HCV}$ 感染的动物模型。虽然已经证明树鼠对 $\mathrm{HCV}$ 易感, 但目前缺乏树舀试验动物的标准品系, 对实 验数据的可重复性及可信度提出了挑战, 因此, 大 部分科学家仍然倾向于使用改造过的传统实验动 物(如小鼠)。正如美国学者 Robert Lanford 所表示, “如果研发花费数百万美元的药物进入临床前的最 后试验阶段, 我们需要寻找一个可以重复的模 型”(Dolgin, 2011)。

丁型肝炎(hepatitis D virus, HDV)模型 丁肝 病毒的复制必需依靠乙肝病毒的辅助, 因此丁肝病
毒感染模型必须以乙肝病毒感染为基础(Chen et al, 2008)。Li et al (1995)建立了树鼠丁型肝炎/乙型肝 炎感染模型, 并证实树鼠的病理变化与黑猩猩的 感染类似, 提示树舀可以建立 HDV 感染的动物 模型。

就目前的研究资料看, 除人和黑猩猩外, 树鼣 是经证实能感染人乙、丙型肝炎病毒的小型哺乳类 动物, 其他动物模型, 如啮齿类动物和禽类动物病 毒性肝炎模型的不足都可以通过树鼣模型来解决。 因此, 树鼠作为肝炎病毒感染的动物模型具有独特 的优势和不可替代的价值。

\subsection{2 耐药细菌感染和败血症模型}

自抗生素发明以来, 大量耐药菌株的出现使人 类又面临新的挑战。感染是目前医院住院病人死亡 的主要原因之一。美国败血症感染 75 万/年, 死 亡 22.5 万(Hotchkiss \& Karl, 2003)。良好的细菌感 染模型能够提供有效的研究途径。目前人们主要用 啮齿类动物, 如大鼠、小鼠建立细菌感染的动物模 型, 但由于种属差异, 在细菌感染发病进程、机制 及药物药效学和毒理学方面与人临床存在较大差 异。如人败血症是由感染引起的全身性炎症反应 (Goldstein et al, 2005), 而传统的败血症动物模型(如 啮齿类)则表现在对内毒素反应的敏感程度和细胞 因子的动态水平等方面(Dyson \& Singer，2009)。Li SA et al (2012)报道了树鼣金黄色葡萄球菌皮肤惣 伤感染模型和绿脓杆菌涤纶补片包埋感染模型, 表 明树鼣能够构建细菌感染模型和评价抗菌药物。而 他们用细菌脂多糖(LPS)建立的树鼠败血症模型表 明, 树鼠对 LPS 的反应跟小鼠有较大差异, 而与人 更为接近, 提示树鼠有望成为更好的败血症模型 ${ }^{2}$ 。 作为低等灵长类, 树鼠有望替代啮齿类更好地模拟 人败血症的发生和病理进程以及药物药效和毒理 反应等, 值得进一步深入研究。

\section{2 精神和神经疾病模型}

\subsection{1 心理应激和抑郁症动物模型}

有机体对伤害性事件的适应性反应称为应激。 应激是临床医学中涉及的一个普遍生物学问题, 可 导致或恶化一系列人类重大疾病。研究显示, 树鼠 是对心理应激最敏感的动物。束缚应激导致树鼠皮 质醇增高, 是非人灵长类和人类的独有特点, 而啮 齿类动物的应激反应则为皮质酮增高(Collins et al,

\footnotetext{
$\overline{2}$ 引自 Li SA, Liu J, Zhang Y. 2012. Tree shrew, a potential experimental animal for sepsis model. 第一届灵长类动物模型学术论坛会议摘要集. 云南, 昆明: p30.
} 
1984)。Fuchs et al (1990)报道社会竞争失败导致树 鼠尿液的裉黑素大幅度增高, 可能与海马糖皮质激 素受体减少有关, 导致应激反应长时间持续 (Jöhren et al，1994)。慢性社会竞争失败的树䳔由于 皮质醇持续增高, 导致海马 CA3 区神经细胞出现萎 缩现象, 这与人类抑郁症患者的海马萎缩现象高度 一致(Magariños et al，1996)。成年神经细胞新生是 20 世纪末最重要的神经科学进展之一, Gould et al (1997) 利用树鼠证明了心理应激能通过 NMDA 受 体影响成年海马神经细胞新生。

由于心理应激是抑郁症的主要诱因之一, 利用 树獡创建应激病因学的抑郁症模型就有可能模拟 人类抑有症的一些重要特征, 而在其他动物 (如啮 齿类)中却难以做到, 因为树鼠、猴子和人类应激系 统的最基本激素均为皮质醇, 而啮齿类则为皮质 酮。Wang et al (2012)详细分析了树鼠作为抑郁症动 物模型的潜在优势, 即除了脑生理结构和竞争失败 后的反应与人相似外, 其昼行习性可以弥补啮齿类 动物夜行习性的不足。利用树鼠进行打斗, 建立社 会竞争失败的抑郁症模型, 打斗失败树鼠表现出和 人相似的抑郁症状(Wang et al, 2011)。Meng et al ${ }^{3}$ 以糖水偏爱和糖水操作式条件反射方法, 建立了反 应快感缺失和动机缺乏的抑郁症树鼠模型。德国 Fuchs \& Flugge (2002)详细研究了打斗挫败树鼠的 生理、脑功能和行为表现, 证明挫败树鼠的行为和 内分泌症状与抑郁症患者相似, 并且可以通过药物 治疗进行改善, 认为树鼠是研究抑郁的适合模型 (Lucassen et al, 2004)。值得注意的是, 应激导致的 树鼣神经肽 $Y$ 的改变与灵长类相似, 与啮齿类则完 全相反(Zambello et al, 2010)。因此, 应激病因学 的抑有症树鼠模型, 可能是模拟人类抑郁症的理想 模型之一, 已经得到了领域同行的认可(Fuchs, 2005; Vollmayr et al, 2007)。

\subsection{2 树鼠近视模型}

目前近视研究主要是利用鸡、猴子和树鼠建立 动物模型。鸡的近视模型最大问题是其亲缘关系和 人相对较远, 眼睛生理结构与和人有很多不同。虽 然猴子与人在眼睛大小和结构上最接近, 但是它们 的实验成本高、周期长, 而且很难区分长时间形觉 剥夺(visual form deprivation)造成的实验性近视眼 是干预的结果, 还是由于从视网膜传递信号到巩膜
引起的。树鼠实验成本低、周期短, 且其眼睛发育 过程与人相似。在形觉剥夺实验中能精确地控制视 觉环境, 得到稳定的结果。可以通过化学物质(如 $\beta$-APN 或者 DPA)诱导 (Norton, 1999), 或形觉剥 夺来建立树鼠近视模型(McBrien \& Norton, 1994;

Norton, 1999)。动物模型能够方便我们研究近视产 生的机理、探索预防和治疗措施。McBrien et al (2012)的研究表明, 短时间戴凸镜或许可以在一定 程度上矫正近视。通过对转录水平和翻译水平的研 究 (Frost \& Norton, 2012; Siegwart \& Norton, 2002), 可更深入地了解近视的机制。由此可见, 树 鼠在近视模型中占有重要地位, 已成为国际公认的 近视动物模型。

\subsection{3 树鼠老年痴呆模型}

Pawlik et al (1999)就报道了老年树鼠存在类似 于人类老年痴呆症病理样的老年斑, 最近 Yamashita (2012) 的报道支持了猕猴和树鼠均有这种人类老 年痴呆症病理样的老年斑。另外，通过对树鼠基因 组的分析, 发现人类与老年痴呆症有关的基因和树 鼠中的直系基因具有较高的同源度(Fan et al, 2012)。显然, 树鼠有可能是创建人类老年痴呆症模 型的另一理想动物。

\section{3 代谢性疾病模型}

Schwaier (1979)报道了高胆固醇饮食可诱发树 鼠生成胆结石, 是胆结石研究合适、方便的模型。

链脲佐菌素(streptozotocin, STZ) 是链球菌产生 的天然化合物, 对哺乳动物的胰岛 $\mathrm{B}$ 细胞有特异毒 性, 被广泛用于诱导 1 型和 2 型糖尿病。Ishiko et al (1997)和 Xian et al (2000)初步报道利用不同浓度 的 STZ 可以诱导树鼠患糖尿病。人类 2 型糖尿病患 者和非人灵长类 2 型糖尿病动物的胰腺具有胰岛 细胞淀粉样沉积, Wagner et al (2001)证实 STZ 诱 导的糖尿病食蟹猴并没有淀粉样沉积症状, 但 Xian et al (2000)用 STZ 诱导的糖尿病树鼠淀粉染色呈阳 性, 是否是胰岛细胞淀粉样沉积需要进一步证实。

$\mathrm{Wu}$ et al (2012) 开展了树鼠糖尿病的动物模型 研究, 多次小剂量 STZ $(60 \sim 80 \mathrm{mg} / \mathrm{kg})$ 能够导致树 鼠糖尿病。注射 STZ 后, 成模树鼠出现明显的多饮、 多食和多尿症状; 持续高血糖、尿糖; 糖耐受明显 受损, 且出现明显糖脂代谢紊乱; 肾功能显著受损; 未出现糖尿病乳酸中毒和高血糖高渗等并发症。

\footnotetext{
${ }^{3}$ 引自孟肖路, 朱宁, 隋南, 祁可可. 2012. 新型树獡社会挫败模型的建立. 第一届灵长类动物模型学术论坛会议摘要集, 云南, 昆明: p 38 .
} 
Zhang et $\mathrm{al}^{4}$ 开展了树舄非酒精性脂肪肝模型 的研究, 高脂、高糖、高胆固醇食物能够诱导树鼠 脂肪肝, 病症与人非酒精性脂肪肝类似, 具有成模 率高、可模拟人疾病进程的特点, 值得进一步深入 研究。

Li \& Zhang (1999)用树鼠开展脑缺血疾病研 究, 已成功建立了光诱导树鼠脑缺血模型。由于树 鼠颅骨很薄, 可通过光照射进行诱导, 使大脑血管 形成血栓, 造成大脑缺血; 加上其大脑发达, 与灵 长类接近, 且经济、容易饲养, 被认为是研究脑血. 管疾病机制的理想动物。

\section{4 癌症模型}

树鼠是比较理想的肿瘤模型。首先, 多个报道 显示树鼠自发各种肿瘤。Elliot et al (1966)报道了第 一例树鼠自发乳腺癌模型, 随后几种其他自发肿瘤 如肝癌(Hofmann et al, 1981)、淋巴瘤 (Brack, 1998) 和肺癌(Brack et al, 1996)等也被报道。Xia et al (2012)鉴定了一例自发性乳腺肿瘤, 该肿瘤与人导 管乳头状瘤很接近, 提示树鼠可以用于建立乳腺肿 瘤模型。其次, 树鼠可以诱发各种癌症。Reddy et al (1976)用黄曲霉毒素诱导树鼠肝癌获得成功。Yan et al (1996)组合乙肝病毒感染和黄曲霉毒素在 52.9\% 的树鼠中成功诱发肝癌, 在树鼠肝癌中检测到 $p 53$ 抑癌基因突变(Park et al, 2000)以及癌基因 Ras 表 达上调 (Su et al，2004)。蛋白质谱分析发现了大量 表达畸变的蛋白(Li et al，2008)。该团队的研究发 现 Oltipraz 可以减少黄曲霉毒素诱发树舄产生肝癌 (Li et al, 2000)。此外, 树鼠还可以诱导肺癌和乳 腺癌。Rao \& Reddy (1980)报道用致癌剂 DHPN 可 以诱导树鼠在 $65 \sim 102$ 周生成肺癌。中科院昆明动 物所陈策实在 2012 年云南昆明第一届灵长类动物 模型学术论坛上, 介绍了用致癌剂 DMBA 组合孕 激素以及慢病毒导入癌基因的方法可以建立树鼠 乳腺肿瘤。

因为肿瘤发生和生理生化有关, 树鼠癌症模型 相对小鼠模型具有诸多优势。例如小鼠诱导的乳腺 癌模型一般为雌激素受体 ER 阴性，而 $60 \% \sim 70 \%$ 的人乳腺癌患者为 $E R$ 阳性, 同时, 小鼠乳腺癌和 人病理组织形态差异较大。另外, 小鼠的乳腺癌转 移多为肺部转移, 而在乳腺癌患者中肿瘤细胞常经
局部淋巴管转移至淋巴结然后多发生骨、脑等部位 转移, 这可能是由于小鼠和人的归巢机制有较大差 异。此外, 已知端粒在癌症发生发展过程中扮演着 很重要的角色。小鼠细胞染色体端粒较长 $(40 \sim 60$ $\mathrm{kb})$, 而人染色体端粒较短 $(10 \mathrm{~kb})$, 因此, 小鼠细胞 易于永生化和转化。由于和人更加类似的进化地 位, 树鼠乳腺癌模型较小鼠模型在生理和病理方面 更加接近人乳腺癌。

肿瘤发生发展与个体精神状态有关，不同的精 神压力会调控肿瘤生长。树鼠在神经系统方面具有 啮齿类无法比拟的优势, 较啮齿类更适用于研究精 神压力与肿瘤的关系。

此外, 免疫系统在肿瘤发生中发挥着重要作 用, 免疫细胞一方面清除肿瘤细胞, 另一方面被肿 瘤细胞利用促进生长和转移。多种肿瘤, 如肝癌、 宫颈癌、鼻咽癌等均与病毒和细菌感染相关。而免 疫系统在不同物种间的差异很大，树獡的免疫系统 与人更加接近 (Fan et al, 2012), 在评价涉及免疫 系统的抗肿瘤药物如单克隆抗体、疫苗和促进机体 免疫力的治疗方法等研究领域更具优势。

虽然树鼠在创制癌症模型方面相对小鼠更具 优势, 但是目前还需要完善树鼠相关的基础研究, 全面了解树鼠与人和鼠的各种组织尤其是神经和 免疫系统的异同, 为创建与人发病机理更加类似的 树鼠癌症模型奠定基础。

\section{4 现存问题和未来展望}

迄今为止, 还没有一种国际上广泛使用的动物 模型由我国科学家创建。近年来, 树鼠作为一种新 型实验动物, 正日益受到国内外的关注。中国科学 院昆明动物研究所于 2012 年 12 月在昆明主办了 “第一届灵长类动物模型学术论坛”研讨会, 会议摘 要的一半是关于树鼣研究的。用树鼠原代肝细胞找 到乙肝和丁肝病毒受体的科学突破(Yan et al, 2012), 也极大地激励着我们去探索树䳔模型的物种 优势。

通过昆明动物所近期对树鼣全基因组、转录组 (Fan et al, 2012)及蛋白组(Li RX et al, 2012)的解析, 我们了解到树鼠与灵长类的遗传特征接近, 这为我 们了解树鼠生物学特性, 发掘其用于疾病模型创

\footnotetext{
${ }^{4}$ 引自: Zhang L, Wu X, Chang Q, Li Y, Liao S, Liang B. 2012. The establishment of tree shrew model of non-alcoholic fatty liver disease. 第一届灵长类动物 模型学术论坛会议摘要集. 云南, 昆明: p65.
} 
制、疾病机理解析和新药创制, 以及回答一些重大 科学问题提供了扎实的科学数据。现有文献报道了 相当多的树䳔基础生物学数据, 但是一些关键数 据, 如树鼣脑组织精细解剖图谱分析等依然缺乏。 而且, 相对于其他成熟的实验动物, 现有的树鼠基 础生物学数据和专有检测试剂远远不够, 而传统的 实验动物如小鼠则存在有大量共享的网上生物信
息资源及商业化的蛋白抗体等。这种信息蒉乏的情 况制约着今后在分子水平上研发人类疾病的树鼠 动物模型, 国内外对于树鼠研究的重视程度和产出 有待大幅提高(图 2)。我们需要系统地了解树舄基因 组、转录组、蛋白质组、神经系统 (脑功能)重、代 谢系统和免疫系统分子基础、基因和蛋白质结构和 功能信息及生物学行为等信息。

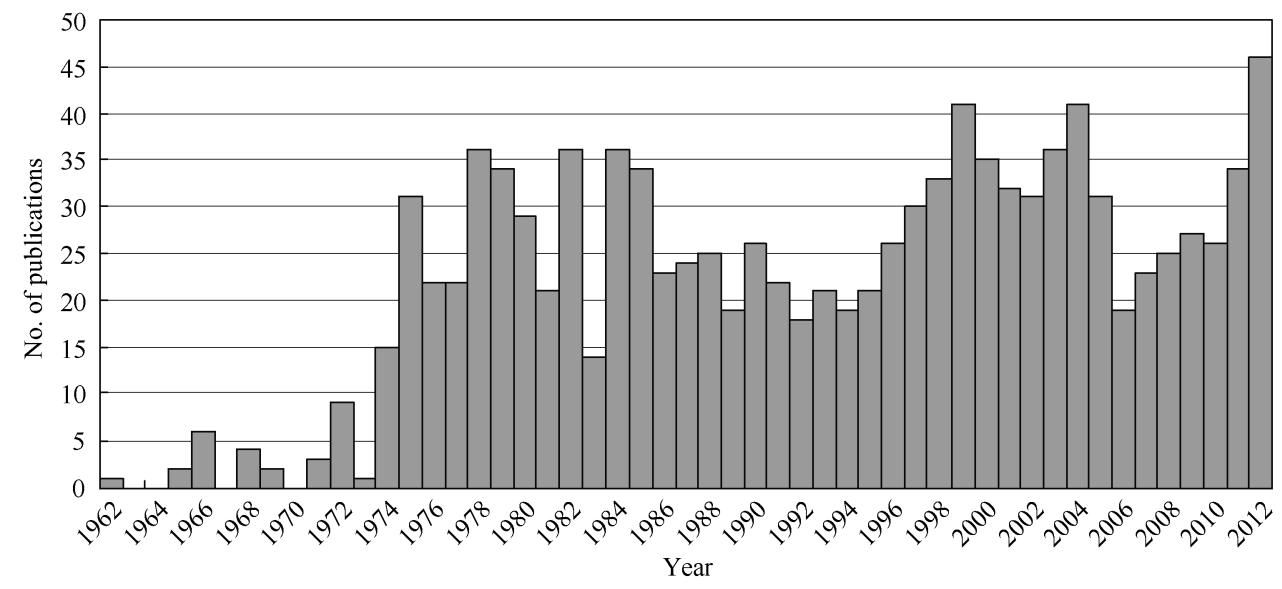

图 2 采用“tree shrews”在 PubMed 数据库中检索的文献数

Figure 2 Number of publications retrieved by key word "tree shrews" through PubMed on Nov. 25, 2012 (1112 publications)

实验动物饲养和繁殖条件的标准化、质量的标 准化、以及检测方法与技术的标准化和规范化都是 规模化应用实验动物进行人类疾病研究和新药研 发的基本必要条件。虽然树鼠已被较多地应用于医 学生物学基础研究中, 也有一些积累和发现, 但迄 今为止, 我们仍然缺乏遗传背景清晰、遗传成分稳 定的种群或品系, 这使得现有研究工作在深度及广 度方面均存在缺陷, 有待进一步提升。建立树鼣纯 品系和相关封闭繁殖群, 尽早完成其实验动物的模 式化和标准化, 并在全国乃至全世界予以推广, 是 目前我们迫切需要解决的一个重大问题。与此同 时, 需要深入阐述树鼣模型在哪些重大生物学问题 和人类疾病研究中具有不可替代的优势, 最终实现

\section{参考文献:}

Adkins RM, Honeycutt RL. 1991. Molecular phylogeny of the superorder archonta. Proc Natl Acad Sci USA, 88(22): 10317-10321.

Amako Y, Tsukiyama-Kohara K, Katsume A, Hirata Y, Sekiguchi S, Tobita Y, Hayashi Y, Hishima T, Funata N, Yonekawa H, Kohara M. 2010. Pathogenesis of hepatitis $\mathrm{C}$ virus infection in Tupaia belangeri. $\mathrm{J}$ Virol, 84(1): 303-311.

Brack M. 1998. Spontaneous tumours in tree shrews (Tupaia belangeri):
“中国制造”的树鼠品系和模型。这些工作的开展和 最终完成, 将彻底解决树獡在疾病机理研究和新药 研发中广泛应用的瓶颈。毫无疑问, 这是一个需要 长时间积累、延续和不断大量投入的系统工作。

致谢: 非常感谢中国科学院昆明动物研究所王 霜女士在文稿的整理中给予的巨大帮助! 同时感谢 郑永唐研究员、赖伆研究员、赵旭东研究员和张华 堂研究员对本文的支持和关注。由于篇幅和写作时 间的局限, 我们对于树舀研究工作的介绍不能作到 面面俱到, 对于文中未能引用的文献, 在此向原作 者致歉。

population studies. J Comp Pathol, 118(4): 301-316.

Brack M, Schwartz P, Heinrichs T, Schultz M, Fuchs E. 1996. Tumors of the respiratory tract observed at the German Primate Center, 1978-1994. J Med Primatol, 25(6): 424-434.

Cao J, Yang, EB, Su JJ, Li Y, Chow P. 2003. The tree shrews: adjuncts and alternatives to primates as models for biomedical research. J Med Primatol, 32(3): 123-130 
Chayama K, Hayes CN, Hiraga N, Abe H, Tsuge M, Imamura M. 2011. Animal model for study of human hepatitis viruses. J Gastroenterol Hepatol, 26(1): 13-18.

Chen J, Dai JJ, Sun XM. 2008. Advances in the use of tree shrews as animal models for hepatitis research. Chn J Com Med, 18(2): 59-62. [陈瑾, 代解杰, 孙晓梅. 2008. 树舄肝炎动物模型的研究进展. 中国比较医学杂志, 18(2): 59-62.]

Chen SY, Xu L, Lü LB, Yao YG. 2011. Genetic diversity and matrilineal structure in Chinese tree shrews inhabiting Kunming, China. Zool Res, 32(1): 17-23. [陈仕毅, 许凌, 吕龙宝, 姚永刚. 2011. 昆明城郊中国树鼠群体线 粒体 DNA 遗传多样性. 动物学研究, 32(1): 17-23.]

Collins PM, Tsang WN, Metzger JM. 1984. Influence of stress on adrenocortical function in the male tree shrew (Tupaia belangeri). Gen Comp Endocrinol, 55(3): 450-457.

Dolgin E. 2011. The murine candidate. Nature, 474(7350): S14-S15.

Dyson A, Singer M. 2009. Animal models of sepsis: why does preclinical efficacy fail to translate to the clinical setting? Crit Care Med, 37(1): S30-S37.

Elliot OS, Elliot MW, Lisco H. 1966. Breast cancer in a tree shrew (Tupaia glis). Nature, 211(5053): 1105.

Fan Y, Huang ZY, Cao CC, Chen CS, Chen YX, Fan DD, He J, Hou HL, Hu L, Hu XT, Jiang XT, Lai R, Lang YS, Liang B, Liao SG, Mu D, Ma YY, Niu YY, Sun XQ, Xia JQ, Xiao J, Xiong ZQ, Xu L, Yang L, Zhang Y, Zhao W, Zhao XD, Zheng YT, Zhou JM, Zhu YB, Zhang GJ, Wang J, Yao YG. 2012. Genome of the Chinese tree shrew, a rising model animal genetically related to primates. Nat Commun, DOI: 10.1038/ncomms 2416

Frost MR, Norton TT. 2012. Alterations in protein expression in tree shrew sclera during development of lens-induced myopia and recovery. Invest Ophthalmol Vis Sci, 53(1): 322-336.

Fuchs E. 2005. Social stress in tree shrews as an animal model of depression: an example of a behavioral model of a CNS disorder. CNS Spectr, 10(3): 182-190.

Fuchs E, Schumacher M. 1990. Psychosocial stress affects pineal function in the tree shrew (Tupaia belangeri). Physiol Behav, 47(4): 713-717.

Fuchs E, Flugge G. 2002. Social stress in tree shrews: effects on physiology, brain function, and behavior of subordinate individuals. Pharmacol Biochem Behav, 73(1): 247-258.

Glebe D, Aliakbari M, Krass P, Knoop EV, Valerius KP, Gerlich WH. 2003. Pre-S1 antigen-dependent infection of Tupaia hepatocyte cultures with human hepatitis B virus. J Virol, 77(17): 9511-9521.

Goldstein B, Giroir B, Randolph A. 2005. International pediatric sepsis consensus conference: definitions for sepsis and organ dysfunction in pediatrics. Pediatr Crit Care Med, 6(1): 2-8.

Gould E, McEwen BS, Tanapat P, Galea LAM, Fuchs E. 1997. Neurogenesis in the dentate gyrus of the adult tree shrew is regulated by psychosocial stress and NMDA receptor activation. $J$ Neurosci, 17(7): 2492-2498.

Hallstrom BM, Janke A. 2010. Mammalian evolution may not be strictly bifurcating. Mol Biol Evol, 27(12): 2804-2816.
Han JB, Zhang GH, Duan Y, Ma JP, Zhang XH, Luo RH, Lü LB, Zheng YT. 2011. Sero-epidemiology of six viruses natural infection in Tupaia belangeri chinensis. Zool Res, 32(1): 11-16. [韩建保, 张高红, 段勇, 马建平, 张喜 鹤, 罗荣华, 吕龙宝, 郑永唐. 2011. 中缅树䳔自然感染六种病毒的血清 流行病学. 动物学研究, 32(1): 11-16.]

Hofmann W, Möller P, Schwaier A, Flügel RM, Zöller L, Darai G. 1981. Malignant tumours in Tupaia (tree shrew). J Med Primatol, 10(2-3): 155-163.

Hotchkiss RS, Karl IE. 2003. Medical progress: the pathophysiology and treatment of sepsis. New Engl J Med, 348(2): 138-150.

Ishiko S, Yoshida A, Mori F, Abiko T, Kitaya N, Kojima M, Saito K. 1997. Early ocular changes in a tree shrew model of diabetes. J Jpn Ophthalmol Soc, 101: 19-23.

Janečka JE, Miller W, Pringle TH, Wiens F, Zitzmann A, Helgen KM, Springer MS, Murphy WJ. 2007. Molecular and genomic data identify the closest living relative of primates. Science, 318(5851): 792-794.

Jöhren O, Flügge G, Fuchs E. 1994. Hippocampal glucocorticoid receptor expression in the tree shrew: regulation by psychosocial conflict. Cell Mol Neurobiol, 14(3): 281-296.

Li QF, Ding MQ, Wang H, Mao Q, Wu CQ, Zheng H, Gu CH, Wang YM. 1995. The infection of hepatitis D virus in adult Tupaia. Nat Med J Chn, 75(10): 611-613. [李奇芬, 丁明权, 王洪, 毛青, 吴纯清, 郑红, 顾长海, 王宇明. 1995. 树獡感染丁型肝炎病毒的实验研究. 中华医学杂志, 75(10): 611-613. ]

Li RX, Xu W, Wang Z, Liang B, Wu JR, Zeng R. 2012. Proteomic characteristics of the liver and skeletal muscle in the Chinese tree shrew (Tupaia belangeri chinensis). Protein Cell, 3(9): 691-700.

Li SA, Lee WH, Zhang Y. 2012. Two bacterial infection models in tree shrew for evaluating the efficacy of antimicrobial agents. Zool Res, 33(1): $1-6$.

Li SQ, Meng Q, Zhang L. 1999. Experimental therapy of a platelet-activating factor antagonist (ginkgolide B) on photochemically induced thrombotic cerebral ischaemia in tree shrews. Clin Exp Pharmacol Physiol, 26(10): 824-825.

Li Y, Su JJ, Yang C, Cao J, Ou C, Liang L, Yang F, Wang Q. 2011. Progress on establishment of tree shrew (Tupaia) chronic infection with HBV in vivo. Zool Res, 32(1): 104-108. [李瑗, 苏建家, 杨春, 曹䩀, 欧超, 梁亮, 杨芳, 王琦. 2011. 乙型肝炎病毒树舀体内慢性感染模型的研究历程和展望. 动物学研究, 32(1): 104-108.]

Li Y, Su JJ, Qin LL, Egner PA, Wang JS, Groopman JD, Kensler TW, Roebuck BD. 2000. Reduction of aflatoxin $\mathrm{B}_{1}$ adduct biomarkers by oltipraz in the tree shrew (Tupaia belangeri chinensis). Cancer Lett, 154(1): 79-83.

Li Y, Qin X, Cui JF, Dai Z, Kang XN, Yue HY, Zhang Y, Su JJ, Cao J, Ou C, Yang C, Duan XX, Yue HF, Liu YK. 2008. Proteome analysis of aflatoxin B1-induced hepatocarcinogenesis in tree shrew (Tupaia belangeri chinensis) and functional identification of candidate protein peroxiredoxin II. Proteomics, 8(7): 1490-1501.

Liang L, Li Y, Yang C, Cao J, Su JJ, Chen MW, Ban KC, Ou C, Duan XX, Yue HF. 2006. Perinatal and young tree shrews infected with human hepatitis B virus. Chn J Zoon, 22(8): 792-795. [梁亮, 李瑗, 杨春, 曹骥, 苏建家，陈茂伟，班克臣，欧超，段小㜆，岳惠芬. 2006. 用人工繁育的 
围生期和幼年树舄感染人乙型肝炎病毒的初步研究. 中国人兽共患病 学报, 22(8): 792-795.]

Lindblad-Toh K, Garber M, Zuk O, Lin MF, Parker BJ, Washietl S, Kheradpour P, Ernst J, Jordan G, Mauceli E, Ward LD, Lowe CB, Holloway AK, Clamp M, Gnerre S, Alfoldi J, Beal K, Chang J, Clawson H, Cuff J, Di Palma F, Fitzgerald S, Flicek P, Guttman M, Hubisz MJ, Jaffe DB, Jungreis I, Kent WJ, Kostka D, Lara M, Martins AL, Massingham T, Moltke I, Raney BJ, Rasmussen MD, Robinson J, Stark A, Vilella AJ, Wen JY, Xie XH, Zody MC, Worley KC, Kovar CL, Muzny DM, Gibbs RA, Warren WC, Mardis ER, Weinstock GM, Wilson RK, Birney E, Margulies EH, Herrero J, Green ED, Haussler D, Siepel A, Goldman N, Pollard KS, Pedersen JS, Lander ES, Kellis M, Inst B, Med BC, Univ W. 2011. A high-resolution map of human evolutionary constraint using 29 mammals. Nature, 478(7370): 476-482.

Liu XH, Yao YG. 2013. Characterization of 12 polymorphic microsatellite markers in the Chinese tree shrew (Tupaia belangeri chinensis). Zool Res, 34 (E2): E63-E69.

Liu Z, Mao Q, Wang YM, Li QF. 1998. An experimental study on infection of hepatitis $C$ virus in adult Tupaiae. $J$ Third Mili Med Univ, 12(6): 472-475. [刘志, 毛青, 王宇明, 李奇芬. 1998. 丙型肝炎病毒感染成年树鼠的实 验研究. 第三军医大学学报, 12(6): 472-475.]

Lucassen PJ, Fuchs E, Czeh B. 2004. Antidepressant treatment with tianeptine reduces apoptosis in the hippocampal dentate gyrus and temporal cortex. Biol Psychiatry, 55(8): 789-796.

Ma XT, Li FL, Jiang HJ, Lee WH, Zhang Y, Du TY. 2011. Detection and comparison of physiological indexes in the wild and laboratory tree shrew. Zool Res, 32(1): 4-10. [马旭通, 李福林, 蒋宏君, 李文辉, 张云, 杜廷义. 2011. 野生和人工繁育树舄部分生理指标检测与比较. 动物学研究, 32(1): 4-10.]

Magariños AM, McEwen BS, Flugge G, Fuchs E. 1996. Chronic psychosocial stress causes apical dendritic atrophy of hippocampal CA3 pyramidal neurons in subordinate tree shrews. $J$ Neurosci, 16(10): 3534-3540.

McBrien NA, Norton TT. 1994. Prevention of collagen crosslinking increases form-deprivation myopia in tree shrew. Exp Eye Res, 59(4): 475-486.

McBrien NA, Arumugam B, Metlapally S. 2012. The effect of daily transient $+4 \mathrm{D}$ positive lens wear on the inhibition of myopia in the tree shrew. Invest Ophth Vis Sci, 53(3): 1593-1601.

Nie WH, Fu BY, O'Brien PC, Wang JH, Su WT, Tanomtong A, Volobouev V, Ferguson-Smith MA, Yang FT. 2008. Flying lemurs-the 'flying tree shrews'? Molecular cytogenetic evidence for a scandentia-dermoptera sister clade. BMC Biol, 6: 18

Norton TT. 1999. Animal models of myopia: learning how vision controls the size of the eye. ILAR J, 40(2): 59-77.

Pang QF, Wan XB, Xu AY, Wang ZM, Wang GX, Zhu BY, Zhang XS. 1981. Hepatitis B virus (HBV) infection in the experimental tree shrews (Abstract). $J$ Med Res, (9): 11-12. [庞其方, 万新邦, 胥爱源, 王祖铭, 王桂香, 朱宝 友, 张新生. 1981. 乙型肝炎病毒 (HBV)感染树舄的实验研究(摘要). 医 学研究通讯, (9): 11-12.]

Park US, Su JJ, Ban KC, Qin LL, Lee EH, Lee YI. 2000. Mutations in the p53 tumor suppressor gene in tree shrew hepatocellular carcinoma associated with hepatitis B virus infection and intake of aflatoxin B1. Gene,
251(1): 73-80.

Pawlik M, Fuchs E, Walker LC, Levy E. 1999. Primate-like amyloid-beta sequence but no cerebral amyloidosis in aged tree shrews. Neurobiol Aging, 20(1): 47-51.

Peng YZ, Ye ZZ, Zou RJ, Wang YX, Tian BP, Ma YY, Shi LM. 1991. Biology of Chinese Tree Shrews [M]. Kunming: Yunnan Science and Technology Press. [彭燕章, 叶智彰, 邹如金, 王应祥, 田保平, 马原野, 施立明. 1991. 树鼠生物学. 昆明: 云南科技出版社.]

Rao MS, Reddy JK. 1980. Carcinogenicity of 2, 2 -dihydroxy-di-npropylnitrosamine in the tree shrew (Tupaia glis): light and electron microscopic features of pulmonary adenomas. $J$ Natl Cancer Inst, 65(4): 835-840.

Reddy JK, Svoboda DJ, Rao MS. 1976. Induction of liver tumors by aflatoxin B1 in the tree shrew (Tupaia glis), a nonhuman primate. Cancer Res, 36(1): 151-160.

Schmitz J, Ohme M, Zischler H. 2000. The complete mitochondrial genome of Tupaia belangeri and the phylogenetic affiliation of scandentia to other eutherian orders. Mol Biol Evol, 17(9): 1334-1343.

Schwaier A. 1979. Tupaias (tree shrews)-a new animal model for gallstone research. Res Exp Med (Berl), 176(1): 15-24.

Shen PQ, Zheng H, Liu RW, Chen LL, Li B, He BL, Li JT, Ben KL, Cao YM, Jiao JL. 2011. Progress and prospect in research on laboratory tree shrew in China. Zool Res, 32 (1): 109-114. [沈培清, 郑红, 刘汝文, 陈丽 玲, 李波, 何保丽, 李进涛, 贲昆龙, 曹篠梅, 角建林. 2011. 中国树舄实 验动物化研究进展和展望. 动物学研究, 32(1): 109-114.]

Siegwart JT, Norton TT. 2002. The time course of changes in mRNA levels in tree shrew sclera during induced myopia and recovery. Invest Ophthalmol Vis Sci, 43(7): 2067-2075.

Su JJ, Yan RQ, Gan YQ, Zhou DN, Huang DR, Huang GH. 1986. A Study of experimental infection by human hepatitis B virus (HBV) in adult tree shrew. Shanghai Lab Anim Sci, 6(4): 193-198. [苏建家, 严瑞琪, 甘友全, 周德南, 黄定瑞, 黄国华. 1986. 人乙型肝炎病毒实验感染成年树鼩的 研究. 上海实验动物科学, 6(4): 193-198.]

Su JJ, Ban KC, Li Y, Qin LL, Wang HY, Yang C, Ou C, Duan XX, Lee YL, Yang RQ. 2004. Alteration of $\mathrm{p} 53$ and $\mathrm{p} 21$ during hepatocarcinogenesis in tree shrews. World J Gastroenterol, 10(24): 3559-3563.

Vollmayr B, Mahlstedt MM, Henn FA. 2007. Neurogenesis and depression: what animal models tell us about the link. Eur Arch Psychiatry Clin Neurosci, 257: 300-303.

Wagner JD, Cline JM, Shadoan MK, Bullock BC, Rankin SE, Cefalu WT. 2001. Naturally occurring and experimental diabetes in cynomolgus monkeys: a comparison of carbohydrate and lipid metabolism and islet pathology. Toxicol Pathol, 29: 142-148.

Walter E, Keist R, Niederost B, Pult I, Blum HE. 1996. Hepatitis B virus infection of tupaia hepatocytes in vitro and in vivo. Hepatology, 24(1): 1-5.

Wang HP, Zhou YX, Yao ZQ, Hong S, Li GY. 1997. Preliminary study of HCV infected Adult tree shrews. J Fourth Mili Med Univ, 18(4): 375-376. [王海平, 周永兴, 姚志强, 洪沙, 李光玉. 1997. 成年树舄实验感染丙型 肝炎病毒的初步研究. 第四军医大学学报, 18(4): 375-376.]

Wang J, Zhou QX, Tian M, Yang YX, Xu L. 2011. Tree shrew models: a 
chronic social defeat model of depression and a one-trial captive conditioning model of learning and memory. Zool Res, 32(1): 24-30.

Wang J, Xu XL, Ding ZY, Mao RR, Zhou QX, Lü LB, Wang LP, Zhang C, Xu L, Yang YX. 2013. Basal physiological indexes in domesticated tree shrews. Zool Res, 34 (in press)

Wang J, Zhou QX, Lü LB, Xu L, Yang YX. 2012. A depression model of social defeat etiology using tree shrews. Zool Res, 33(1): 92-98. [王静, 周 启心, 吕龙宝, 徐林, 杨跃雄. 2012. 社会竞争失败病因学的抑郁症树鼠 模型. 动物学研究, 33(1): 92-98.]

Wang XX, Li JX, Wang WG, Sun XM, He CY, Dai JJ. 2011. Preliminary investigation of viruses to the wild tree shrews (Tupaia belangeri chinese). Zool Res, 32(1): 66-69. [王新兴, 李婧潇, 王文广, 孙晓梅, 何春艳, 代解 杰. 2011. 野生中缅䳔病毒携带情况的初步调查. 动物学研究, 32(1): 66-69.]

Wang YX. 1987. Taxonomic research on Burma-Chinese tree shrew, Tupaia belangeri (Wagner), from Southern China. Zool Res, 8(3): 213-230. [王应祥. 1987. 中国树舄的分类研究. 动物学研究, 8(3): 213-230.]

Wu X, Chang Q, Zhang Y, Zou X, Chen L, Zhang L, Liang B. 2013. Relationships between body weight, fasting blood glucose, sex, and age in tree shrews (Tupaia belangeri chinensis). J Anim Physiol Anim Nutr, in press.

Xia H, Wang C, Zhang H, He B, Jiao J, Chen C. 2012. Characterization of spontaneous breast tumor in tree shrews (Tupaia belangeri chinenesis). Zool Res, 33(1): 55-59.

Xian S, Huang S, Su JJ, Qin YF, Ou C, Luo ZJ, Wei MY. 2000. A study on experimental diabetes animal models in tree shrews induced by streptozotocin. J Guangxi Med Univ, 17(6): 945-948. [洗苏, 黄松, 苏建家, 秦映芬, 欧超, 罗佐杰, 韦敏怡. 2000. 链腿佐菌素诱导树㔠糖尿病动物 模型研究. 广西医科大学学报, 17(6): 945-948.]

Xie ZC, Riezu-Boj JI, Lasarte JJ, Guillen J, Su JH, Civeira MP, Prieto J. 1998. Transmission of hepatitis C virus infection to tree shrews. Virology, 244(2): 513-520.

Xu L. 2011. Aniaml models of human diseases. Zool Res, 32(1): 1-3. [徐林. 2011. 人类疾病的动物模型. 动物学研究, 32(1): 1-3.]

Xu L, Fan Y, Jiang XL, Yao YG. 2013. Molecular evidence on the phylogenetic position of tree shrew (Tupia belangeri). Zool Res, 34 (2):70-76. [许凌, 范宇, 蒋学龙, 姚永刚. 2013. 树䳔进化分类地位的分 子证据. 动物学研究, 34 (2):70-76.]
Xu L, Chen SY, Nie WH, Jiang XL, Yao YG. 2012. Evaluating the phylogenetic position of Chinese Tree Shrew (Tupaia belangeri chinensis) based on complete mitochondrial genome: implication for using tree shrew as an alternative experimental animal to primates in biomedical research. $J$ Genet Genomics, 39(3): 131-137.

Yamashita A, Fuchs E, Taira M, Yamamoto K, Hayashi M. 2012. Somatostatin-immunoreactive senile plaque-like structures in the frontal cortex and nucleus accumbens of aged tree shrews and Japanese macaques. J Med Primat, 41(3): 147-157.

Yan H, Zhong GC, Xu GW, He WH, Jing ZY, Gao ZC, Huang Y, Qi YH, Peng B, Wang HM, Fu L, Song M, Chen P, Gao WQ, Ren BJ, Sun YY, Cai T, Feng XF, Sui JH, Li WH. 2012. Sodium taurocholate cotransporting polypeptide is a functional receptor for human hepatitis B and D virus. Elife, 1: $\mathrm{e} 00049$.

Yan RQ, Su JJ, Chen ZY, Liu YG, Gan YQ, Zhou DN. 1984. A preliminary study on experimental infection of human hepatitis B virus in adult tree shrews. J Guangxi Med Univ, 1(1): 10-15. [严瑞其, 苏建家, 陈志英, 刘由 庚, 甘友全, 周德南. 1984. 人乙型肝炎病毒实验感染成年树舀的初步 研究. 广西医学院学报, 1(1): 10-15.]

Yan RQ, Su JJ, Huang DR, Gan YC, Yang C, Huang GH. 1996. Human hepatitis B virus and hepatocellular carcinoma. II. Experimental induction of hepatocellular carcinoma in tree shrews exposed to hepatitis B virus and aflatoxin B1. J Cancer Res Clin Oncol, 122(5): 289-295.

Yang F, Cao J, Zhang JJ, Wang Q, Su JJ, Yang C, Ou C, Shi JL, Wang DP, Li Y. 2009. Long-term observation of hepatitis B virus (HBV) replication in new-born tree shrews inoculated with HBV. Chn J Hepatol, 17(8): 580-584. [杨芳, 曹䩀, 张晶晶, 王琦, 苏建家, 杨春, 欧超, 史俊林, 汪多平, 李 瑗. 2009. 新生期树舄接种人乙型肝炎病毒的长期实验观察. 中华肝脏 病杂志, 17(8): 580-584.]

Zambello E, Fuchs E, Abumaria N, Rygula R, Domenici E, Caberlotto L. 2010. Chronic psychosocial stress alters NPY system: different effects in rat and tree shrew. Prog Neuropsychopharmacol Biol Psychiatry, 34(1): 122-130.

Zhan MY, Liu CX, Li CM, Zhang WY, Zhu C, Pang QF, Zhao TX, Wang CA, Wang JL, Yu CY, Li SF, Tong ZG, Lin ZH, Niu JQ. 1981. A preliminary study of hepatitis A virus in Chinese Tupaia. Acta Acad Med Sin, 3(3): 148-152. [詹美云, 刘崇相, 李成明, 张文英, 朱纯, 庞其方, 赵同兴, 王 长安, 王金利, 余昌晏, 李寿复, 佟智功, 林尊慧, 牛京勤. 1981. 甲型 肝炎病毒感染树舄的初步研究. 中国医学科学院学报, 3(3): 148-152.] 